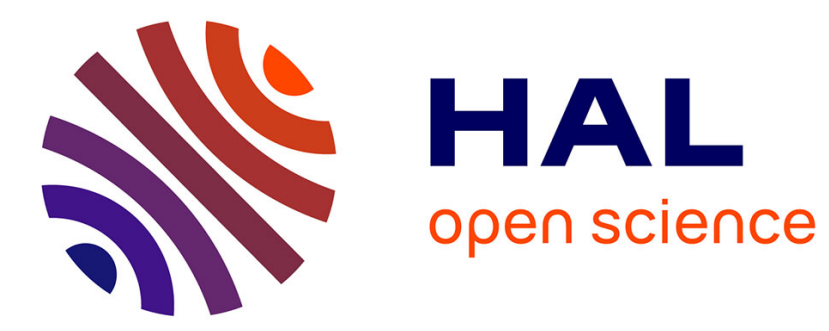

\title{
Définition de la perméabilité équivalente des massifs fracturés par des méthodes d'homogénéisation
}

\author{
Ahmad Pouya, Courtois Alexis
}

\section{To cite this version:}

Ahmad Pouya, Courtois Alexis. Définition de la perméabilité équivalente des massifs fracturés par des méthodes d'homogénéisation. Comptes rendus de l'Académie des sciences. Série IIa, Sciences de la terre et des planètes, 2002, 334, pp.975-979. 10.1016/S1631-0713(02)01839-4 hal-00578015

\section{HAL Id: hal-00578015 \\ https://hal.science/hal-00578015}

Submitted on 18 Mar 2011

HAL is a multi-disciplinary open access archive for the deposit and dissemination of scientific research documents, whether they are published or not. The documents may come from teaching and research institutions in France or abroad, or from public or private research centers.
L'archive ouverte pluridisciplinaire HAL, est destinée au dépôt et à la diffusion de documents scientifiques de niveau recherche, publiés ou non, émanant des établissements d'enseignement et de recherche français ou étrangers, des laboratoires publics ou privés. 
C. R. Geoscience 334 (2002) 975-979

Comptes-Rendus Académie des Sciences

Géosciences de surface / Surface Geosciences

(Hydrologie-Hydrogéologie / Hydrology-Hydrogeology)

\title{
Définition de la perméabilité équivalente des massifs fracturés par des méthodes d'homogénéisation
}

\section{Definition of the permeability of fractured rock masses by homogenization methods}

\author{
Ahmad Pouya $^{\mathrm{a}}$, Alexis Courtois ${ }^{\mathrm{b}}$ \\ ${ }^{\text {a }}$ G3S - Ecole Polytechnique - 91128 - Palaiseau Cedex - France \\ ${ }^{\mathrm{b}}$ EDF R\&D- Site des Renardières, Ecuelles, 77818 - Moret-sur-Loing, France
}

\section{Correspondant principal :}

Ahmad POUYA : téléphone : 01.69.33.33.86, fax : 01.69.33.30.28

courriel : ahmad.pouya@enpc.fr

\begin{abstract}
In the methods used for determination of the hydraulic behaviour at large scale of fractured rock masses, based on the simulation of flow in a fracture network, the mean flux and mean pressure gradient in the network are not rigorously determined. A method is given for deriving these quantities, in a heterogeneous permeable block, from pressure and flux values on the boundary of the block. A block conductivity tensor is then defined based on the condition of linear variation of the pressure on the boundary of the block. It is shown that this conductivity tensor is symmetric and positive definite. An example of application to a model of fractured medium is given.

permeability /heterogeneity / fracture networks / homogeneization

Résumé - Dans les méthodes utilisées pour déterminer le comportement hydraulique à grande échelle des massifs fracturés, passant par la simulation de l'écoulement dans un réseau des fractures, le gradient moyen de pression et le flux moyen dans le réseau ne sont pas calculés de manière rigoureuse. On donne une méthode de détermination de ces quantités moyennes dans un milieu de perméabilité hétérogène à partir des valeurs de pression et de flux sur le contour du domaine étudié. On définit ensuite un tenseur de conductivité hydraulique moyenne du domaine en partant des conditions de pression variant linéairement sur le contour. On montre que ce tenseur est symétrique et défini positif. Un exemple d'application à un modèle de milieu fracturé est donné.
\end{abstract}




\section{Introduction}

Une méthode courante de détermination des propriétés hydrauliques à grande échelle des massifs fracturés consiste à simuler l'écoulement dans un réseau de fractures, et essayer d'établir des relations entre le gradient moyen de pression et le flux moyen dans ce réseau [1],[2],[3]. Cette méthode, dite directe [4], s'inscrit dans le cadre plus général des méthodes dites non locales de détermination de la perméabilité effective des milieux hétérogènes [5],[6],[7],[8]. Dans ces méthodes, le flux moyen $\overrightarrow{\mathrm{Q}}$ et le gradient moyen de pression $\overrightarrow{\mathrm{G}}$ sont définis explicitement [9] ou implicitement par les relations (3) données ci-après. Mais une analyse des méthodes de calcul adoptées dans ces travaux révèle une certaine difficulté à calculer ces grandeurs moyennes. La difficulté réside dans le fait que les moyennes sont définies sur le volume tandis que, dans les simulations numériques, les valeurs auxquelles on accède plus facilement, et parfois exclusivement, sont les valeurs aux frontières des différentes grandeurs.

Pour illustrer ces difficultés, considérons l'exemple du travail de Long et al. [1] sur le calcul de la perméabilité équivalente des massifs fracturés. Ces auteurs considèrent un domaine carré (ou rectangulaire), comprenant un réseau de fractures, et simulent l'écoulement dans ce réseau en appliquant un gradient de pression parallèle à un de ses cotés du domaine (conditions de pression linéaire au contour [5]). Ils reconnaissant la difficulté de calculer la valeur moyenne du flux dans le volume du fait que le flux est fortement orienté dans les fractures et qu'il est nul en dehors des fractures. Ils calculent pur cela le débit sur les cotés orthogonaux à la direction du gradient. Mais la difficulté subsiste encore : le débit entrant sur un de ces cotés n'est pas égal à celui sortant de l'autre, car une partie du débit peut sortir des cotés latéraux. Cela pose alors, comme le soulignent les auteurs, un problème pour calculer la perméabilité directionnelle dans la direction du gradient. Pour remédier à cette difficulté, Cacas et al. [3] apportent une légère modification à cette méthode en imposant une condition de flux nul aux cotés parallèles à la direction du gradient (conditions de perméamètre [5]). Mais dans ce cas, la pression sur ces côtés n'est plus contrôlée et cela pose le problème du calcul du gradient moyen de pression dans le domaine.

Le calcul des termes non diagonaux du tenseur de perméabilité se heurtent à la même difficulté dans la méthode de Long et al. En effet, en notant x la direction du gradient, si les débit sur les deux cotés parallèles à $\mathrm{x}$ étaient égaux, ils permettraient de calculer $\mathrm{K}_{\mathrm{xy}}$. Ce n'est pas le cas. Par ailleurs, en imposant le même gradient dans la direction de y, on trouve pas le même débit sur les cotés parallèles à y, ce qui vaut dire qu'on trouve pas par cette méthode la même valeur pour $\mathrm{Kxy}$ et $\mathrm{K}_{\mathrm{yx}}$. La question de la symétrie du tenseur de perméabilité reste donc en suspens.

La méthode Long et al. [1] consiste en effet à calculer uniquement la perméabilité dans la direction du gradient, noté $\mathrm{K}_{\mathrm{g}}$, et ensuite, faire des rotations sur le domaine carré par rapport au réseau des fractures pour obtenir le diagramme polaire de $\mathrm{Kg}$ pour diiférentes directions. Le critère adopté alors pour juger si le réseau peut être assimilé à un milieu poreux est que le diagramme polaire $1 / \sqrt{\mathrm{K}_{\mathrm{g}}}$ s'approche d'une ellipse symétrique par 
rapport à l'origine. Il faut noter que cette symétrie signifie uniquement l'égalité des débits entrant et sortant par les cotés opposés. Quant à la symétrie du tenseur de conductivité hydraulique du réseau, cette méthode ne permet pas d'en juger. En effet, même pour un tenseur $\mathbf{K}$ non symétrique, en notant $K_{g}=K_{i j} n_{i} n_{j}$ ou $\vec{n}$ est un vecteur unitaire, et en traçant le digramme de $1 / \sqrt{\mathrm{K}_{\mathrm{g}}}$ en fonction de la direction de $\overrightarrow{\mathrm{n}}$, on trouve une ellipse.

La question de la symétrie du tenseur de perméabilité effective du milieux fracturé reste en suspens. On ne sait pas si le manque de symétrie trouvée dans les méthodes numériques est due aux imprécisions de ces méthodes, ou si elle découle de la nature même des propriétés de conductivité hydraulique du milieu fracturé. Le fait que, comme nous l'avons signalé ci-dessus, le tenseur de conductivité hydraulique ne soit pas rigoureusement défini (car la relation entre n'est pas unique) est une difficulté supplémentaire qui empêche que des propriétés telles que la pertinence ou la symétrie de ce tenseur.

Le travail de Pouya et Courtois (200 ?) a apporté des réponses à une partie de ces questions. Ces auteurs ont d'abord démontré que dans les méthodes numériques cités cidessus, les méthodes de calculs des valeurs moyennes de $\mathrm{Q}$ et de $\mathrm{G}$ à partir des données de la frontière ne sont pas correctes. Ils ont données les formules corrects à partir d'un traitement mathématique rigoureuse. Ensuite, ils ont défini un tenseur de conductivité hydraulique d'un milieu fracturé, correspondant aux conditions de pression linéaire au contour, et ont montré que ce tenseur est bien symétrique et défini-positive.

Dans ce travail nous allons compléter le travail précédent en montrant qu'on peut également définir un tenseur de conductivité hydraulique du bloc correspondant aux conditions de perméamètre. Qui est également symétrique et défini positif. Nous allons ensuite comparer ces deux tenseurs et montrer que les conditions de perméamètre conduisent à des valeurs inférieurs de perméabilité par rapport aux conditions de pression linéaire au contour.

Cette partie du travail, consiste en des traitement mathématiques rigoureux, et valables aussi bien pour des écoulements 3D que 2D. nous étudierons les propriétés. Notre définition et notre démarche s'inspirent des méthodes générales d'homogénéisation du comportement mécanique des matériaux hétérogènes [10].

A cette partie succède une seconde partie qui, moyennant quelques approximations, permet de proposer une méthode de calcul numérique simple du tenseur de perméabilité 3D d'un milieux fracturé à partir des calculs en 2D.

Enfin suit une troisième partie qui consiste en une illustration de ces méthodes par application à un cas réel : le massif --- pour le Tunnel Maurienne-Ambin. 


\section{Position du problème}

Considérons un corps perméable hétérogène occupant un domaine $\Omega$ dans l'espace. Ce corps est le siège d'un écoulement de fluide sous l'effet de pressions ou de flux imposés sur son contour $\partial \Omega$. On suppose qu'il est constitué de matériaux de perméabilités différentes caractérisés chacun par un tenseur de perméabilité noté $\mathbf{k}$ symétrique et défini positif. En tout point de $\Omega$, caractérisé par son vecteur position $\overrightarrow{\mathrm{x}}$, l'écoulement a lieu suivant la loi de Darcy :

$$
\forall \vec{x} \in \Omega ; \quad \vec{q}(\vec{x})=-\mathbf{k}(\vec{x}) \cdot \vec{\nabla} \mathrm{p}(\vec{x})
$$

où $\vec{q}(\vec{x})$ est le flux au point $\vec{x}$ et $\vec{\nabla} p(\vec{x})$ et le gradient de pression en ce point. L'équation de conservation de la masse s'écrit :

$$
\forall \vec{x} \in \Omega ; \quad \operatorname{div} \overrightarrow{\mathrm{q}}(\overrightarrow{\mathrm{x}})=0
$$

Les champs $\mathrm{p}$ et $\overrightarrow{\mathrm{q}}$, solutions du problème d'écoulement, doivent vérifier ces deux équations et les conditions aux limites de flux ou de pression sur $\partial \Omega$. On définit le flux moyen et le gradient moyen de pression dans le corps par les relations suivantes dans lesquelles $\mathrm{V}$ est le volume du domaine $\Omega$ :

$$
\overrightarrow{\mathrm{Q}}=\frac{1}{\mathrm{~V}} \int_{\Omega} \overrightarrow{\mathrm{q}}(\overrightarrow{\mathrm{x}}) \mathrm{dv} \quad, \quad \overrightarrow{\mathrm{G}}=\frac{1}{\mathrm{~V}} \int_{\Omega} \vec{\nabla} \mathrm{p}(\overrightarrow{\mathrm{x}}) \mathrm{dv}
$$

\section{Expression des moyennes volumiques en fonction des valeurs aux frontières}

Pour une fonction $\mathrm{f}$ quelconque, on peut écrire l'identité mathématique suivante (formule de Green) dans laquelle $\vec{n}$ représente le vecteur unitaire sortant et $\mathrm{dS}$, l'élément de surface sur $\partial \Omega$ :

$$
\int_{\Omega} \partial_{\mathrm{i}} \mathrm{f}(\overrightarrow{\mathrm{x}}) \mathrm{dv}=\int_{\partial \Omega} \mathrm{f}(\overrightarrow{\mathrm{x}}) \mathrm{n}_{\mathrm{i}}(\overrightarrow{\mathrm{x}}) \mathrm{dS}
$$

En prenant pour f la pression, on trouve que la moyenne du gradient de pression peut être écrite sous la forme suivante :

$$
\overrightarrow{\mathrm{G}}=\frac{1}{\mathrm{~V}} \int_{\partial \Omega} \mathrm{p}(\overrightarrow{\mathrm{x}}) \overrightarrow{\mathrm{n}}(\overrightarrow{\mathrm{x}}) \mathrm{d} S
$$

En utilisant $\partial_{\mathrm{i}} \mathrm{x}_{\mathrm{j}}=\delta_{\mathrm{ij}}$ (coordonnées cartésiennes) et la conservation de la masse, $\partial_{\mathrm{i}} \mathrm{q}_{\mathrm{i}}=0$, on peut écrire : $\partial_{\mathrm{i}}\left(\mathrm{x}_{\mathrm{j}} \mathrm{q}_{\mathrm{i}}\right)=\left(\partial_{\mathrm{i}} \mathrm{x}_{\mathrm{j}}\right) \mathrm{q}_{\mathrm{i}}+\mathrm{x}_{\mathrm{j}} \partial_{\mathrm{i}} \mathrm{q}_{\mathrm{i}}=\delta_{\mathrm{ij}} \mathrm{q}_{\mathrm{j}}=\mathrm{q}_{\mathrm{i}}$. En remplaçant $\mathrm{q}_{\mathrm{i}}$ dans (3) par $\partial_{\mathrm{i}}\left(\mathrm{x}_{\mathrm{j}} \mathrm{q}_{\mathrm{i}}\right)$ et utilisant $(4)$, on trouve l'expression suivante pour le flux moyen :

$$
\overrightarrow{\mathrm{Q}}=\frac{1}{\mathrm{~V}} \int_{\partial \Omega}(\overrightarrow{\mathrm{q}} \cdot \overrightarrow{\mathrm{n}}) \overrightarrow{\mathrm{x}} \mathrm{dS}
$$

Les formules (5) et (6) permettent de calculer les moyennes volumiques du gradient de pression et du flux à l'aide des pressions et du flux au contour pour une forme du domaine et des conditions aux limites quelconques.

En général, dans les méthodes directes ou non locales, essentiellement deux types de conditions aux limites sont considérés. Les conditions de perméamètre supposent une forme rectangulaire (dans le cas bidimensionnel) pour le domaine, dont deux cotés parallèles sont soumis à des pressions constantes et les deux autres, à un flux sortant $\operatorname{nul}(\overrightarrow{\mathrm{q}} \cdot \overrightarrow{\mathrm{n}}=0)$. Les conditions de pression linéaire au contour sont définies par : 


$$
\forall \overrightarrow{\mathrm{x}} \in \partial \Omega ; \quad \mathrm{p}(\overrightarrow{\mathrm{x}})=\overrightarrow{\mathrm{A}} \cdot \overrightarrow{\mathrm{x}}+\mathrm{P}
$$

où $\overrightarrow{\mathrm{A}}$ est un vecteur constant et $\mathrm{P}$ un scalaire constant.

Contrairement à ce qui est supposé dans les travaux utilisant les conditions de perméamètre, la formule (5) montre que sous ces conditions, la moyenne du gradient de pression ne peut être déduite des seules valeurs de la pression sur les cotés à pression imposée. La formule (6) permet en revanche de montrer que, sous ces conditions, la moyenne volumique du flux est bien égale à la moyenne surfacique du flux sur les cotés à pression imposée, comme c'est souvent admis dans ces travaux.

Considérons maintenant le cas des conditions de pression linéaire au contour. La formule (6) montre que dans ce cas, où le flux n'est (imposé) nul sur aucun coté, le flux moyen ne peut se déduire de la moyenne surfacique sur un seul des côtés. Ceci est l'origine de beaucoup de difficultés dans les travaux [1] utilisant ces conditions.

En prenant dans (4) pour $\mathrm{f}$ une constante non nulle, et ensuite, en prenant pour $\mathrm{f}$ la composante $\mathrm{x}_{\mathrm{j}}$ du vecteur position $\overrightarrow{\mathrm{x}}$, sachant que $\partial_{\mathrm{i}} \mathrm{x}_{\mathrm{j}}=\delta_{\mathrm{ij}}$, on établit successivement les deux identités suivantes :

$$
\int_{\partial \Omega} \vec{n} \mathrm{~d} S=0, \quad \int_{\partial \Omega} \mathrm{x}_{\mathrm{i}} \mathrm{n}_{\mathrm{j}} \mathrm{dS}=\mathrm{V} \delta_{\mathrm{ij}}
$$

En reportant l'expression (7) de $\mathrm{p}(\overrightarrow{\mathrm{x}})$ dans (5) et en utilisant les identités (8), on trouve :

$$
\overrightarrow{\mathrm{G}}=\overrightarrow{\mathrm{A}}
$$

Ce résultat, admis en général dans les travaux utilisant des conditions de pression linéaire au contour sur des domaines rectangulaires avec $\overrightarrow{\mathrm{A}}$ parallèle à un des côtés, se trouve ici établi pour un domaine de forme quelconque.

\section{Tenseur de conductivité hydraulique}

Pour un domaine $\Omega$ fixé, avec une distribution fixée de $\mathbf{k}(\overrightarrow{\mathrm{x}})$ dans $\Omega$, le champ de pression $\mathrm{p}(\overrightarrow{\mathrm{x}})$ solution du problème d'écoulement avec les conditions aux limites de pression linéaire au contour avec les données ( $\vec{A}, P)$ est défini par l'équation (7) et l'équation suivante :

$$
\forall \overrightarrow{\mathrm{x}} \in \Omega ; \quad \operatorname{div}[\mathbf{k}(\overrightarrow{\mathrm{x}}) \cdot \vec{\nabla} \mathrm{p}(\overrightarrow{\mathrm{x}})]=0
$$

En remarquant que si le champ $\mathrm{p}(\overrightarrow{\mathrm{x}})$ est solution de ce problème pour les données $(\vec{A}, P)$, et c est une constante quelconque, alors le champ $p(\vec{x})+c$ est solution du même problème pour les données $(\vec{A}, P+c)$, et le fait que $\vec{\nabla}(p(\vec{x})+c)=\vec{\nabla} p(\vec{x})$, on déduit que le champ de gradient de pression, et donc le champ de flux donné par $\vec{q}(\vec{x})=$ $\mathbf{k}(\overrightarrow{\mathrm{x}}) \cdot \vec{\nabla} \mathrm{p}(\overrightarrow{\mathrm{x}})$, ainsi que sa moyenne volumique $\overrightarrow{\mathrm{Q}}$, sont indépendants de la constate c, et donc de la constate $\mathrm{P}$, et ne dépendent que de $\overrightarrow{\mathrm{A}}$ (et des données intrinsèques qui sont la géométrie de $\Omega$ et la distribution $\mathbf{k}(\overrightarrow{\mathrm{x}})$ ). Considérons maintenant deux vecteurs $\overrightarrow{\mathrm{A}}_{1}$ et $\overrightarrow{\mathrm{A}}_{2}$ et notons respectivement $\mathrm{p}_{1}(\overrightarrow{\mathrm{x}})$ et $\mathrm{p}_{2}(\overrightarrow{\mathrm{x}})$, $\overrightarrow{\mathrm{q}}_{1}(\overrightarrow{\mathrm{x}})$ et $\overrightarrow{\mathrm{q}}_{2}(\overrightarrow{\mathrm{x}})$ et $\overrightarrow{\mathrm{Q}}_{1}$ et $\vec{Q}_{2}$ les champs 
de pression et de flux et les flux moyens associés aux conditions aux limites $\left(\vec{A}_{1}, 0\right)$ et $\left(\vec{A}_{2}, 0\right)$. On vérifie aisément que le champ $\mathrm{p}(\overrightarrow{\mathrm{x}})=\lambda_{1} \mathrm{p}_{1}(\overrightarrow{\mathrm{x}})+\lambda_{2} \mathrm{p}_{2}(\overrightarrow{\mathrm{x}})$ où $\lambda_{1}$ et $\lambda_{2}$ sont deux constantes quelconques, est solution du problème d'écoulement avec les conditions aux limites $(\vec{A}, 0)$, où $\vec{A}=\lambda_{1} \vec{A}_{1}+\lambda_{2} \vec{A}_{2}$. On en déduit que le flux $\vec{Q}$ associé à $\lambda_{1} \vec{A}_{1}+\lambda_{2} \vec{A}_{2}$ est égal à $\lambda_{1} \vec{Q}_{1}+\lambda_{2} \vec{Q}_{2}$, ce qui veut dire que $\vec{Q}$ est une fonction linéaire de $\vec{A}$. Ce résultat s'écrit sous la forme :

$$
\overrightarrow{\mathrm{Q}}=-\mathbf{K}_{\Omega} \cdot \overrightarrow{\mathrm{A}}
$$

Le tenseur $\mathbf{K}_{\Omega}$ est une caractéristique intrinsèque de $\Omega$ ne dépendant que de sa géométrie et de la distribution $\mathbf{k}(\vec{x})$. Considérons maintenant deux champ de pressions $p(\vec{x})$ et $\mathrm{p}^{\prime}(\overrightarrow{\mathrm{x}})$ résultant des conditions aux limites de pression linéaire au contour avec les données respectivement $(\vec{A}, P)$ et $\left(\vec{A}^{\prime}, P^{\prime}\right)$, et notons $\vec{q}$ et $\vec{q}^{\prime}$ les champs de flux correspondants. En tout point de $\Omega$, la relation $\vec{q}^{\prime}=-\mathbf{k} . \vec{\nabla} \mathrm{p}^{\prime}$ et la conservation de la masse, $\partial_{\mathrm{i}} \mathrm{q}_{\mathrm{i}}^{\prime}=0$, permettent d'écrire :

$$
-\vec{\nabla} \text { p.k. } \vec{\nabla} \mathrm{p}^{\prime}=\vec{\nabla} \mathrm{p} \cdot \overrightarrow{\mathrm{q}}^{\prime}=\left(\partial_{\mathrm{i}} \mathrm{p}\right) \mathrm{q}_{\mathrm{i}}^{\prime}=\partial_{\mathrm{i}}\left(\mathrm{pq}_{\mathrm{i}}^{\prime}\right)-\mathrm{p} \partial_{\mathrm{i}} \mathrm{q}_{\mathrm{i}}^{\prime}=\partial_{\mathrm{i}}\left(\mathrm{pq}_{\mathrm{i}}^{\prime}\right)
$$

En intégrant sur $\Omega$, et en appliquant (4) au second membre, on trouve :

$$
-\frac{1}{\mathrm{~V}} \int_{\Omega} \vec{\nabla} \mathrm{p} \cdot \mathbf{k} \cdot \vec{\nabla} \mathrm{p}^{\prime} \mathrm{dv}=\frac{1}{\mathrm{~V}} \int_{\partial \Omega} \mathrm{p} \overrightarrow{\mathrm{q}}^{\prime} \cdot \overrightarrow{\mathrm{n}} \mathrm{dS}
$$

Or, sur $\partial \Omega$, on a $\mathrm{p}(\overrightarrow{\mathrm{x}})=\overrightarrow{\mathrm{A}} \cdot \overrightarrow{\mathrm{x}}+\mathrm{P}$. En reportant cette expression dans le second membre de la relation ci-dessus on trouve :

$$
-\frac{1}{\mathrm{~V}} \int_{\Omega} \vec{\nabla} \mathrm{p} \cdot \mathbf{k} \cdot \vec{\nabla} \mathrm{p}^{\prime} \mathrm{dv}=\frac{1}{\mathrm{~V}} \int_{\partial \Omega}(\overrightarrow{\mathrm{A}} \cdot \overrightarrow{\mathrm{x}}+\mathrm{P}) \overrightarrow{\mathrm{q}}^{\prime} \cdot \overrightarrow{\mathrm{n}} \mathrm{d} S=\overrightarrow{\mathrm{A}} \cdot\left(\frac{1}{\mathrm{~V}} \int_{\partial \Omega}\left(\overrightarrow{\mathrm{q}}^{\prime} \cdot \overrightarrow{\mathrm{n}}\right) \overrightarrow{\mathrm{x}} \mathrm{d} S\right)+\frac{\mathrm{P}}{\mathrm{V}} \int_{\partial \Omega} \overrightarrow{\mathrm{q}}^{\prime} \cdot \overrightarrow{\mathrm{n}} \mathrm{d} S
$$

Dans le dernier membre de ces égalités, $\int_{\partial \Omega} \vec{q}^{\prime} \cdot \vec{n} \mathrm{~d} S=0$ du fait de la conservation de la masse, et le terme entre parenthèse est, d'après (6) et (11), égal à $-\mathbf{K}_{\Omega} \cdot \vec{A}^{\prime}$. On peut donc écrire :

$$
\frac{1}{\mathrm{~V}} \int_{\Omega} \vec{\nabla} \mathrm{p} \cdot \mathbf{k} \cdot \vec{\nabla} \mathrm{p}^{\prime} \mathrm{dv}=\overrightarrow{\mathrm{A}} \cdot \mathbf{K}_{\Omega} \cdot \overrightarrow{\mathrm{A}}^{\prime}
$$

Or, du fait de la symétrie de $\mathbf{k}$, on peut changer le rôle de $\mathrm{p}$ et $\mathrm{p}^{\prime}$ au premier membre de cette égalité. Il en donc de même en ce qui concerne $\vec{A}$ et $\vec{A}^{\prime}$ au second membre. On en déduit que $\mathbf{K}_{\Omega}$ est symétrique.

Ce résultat contredit celui de [1] rapporté dans [5] suivant lequel «les conditions aux limites uniformes (variant linéairement sur le contour) ne produisent pas de perméabilité symétrique ». La différence résulte du fait que, comme nous l'avons expliqué ci-dessus, le flux moyen n'est pas correctement calculé dans [1].

Dans le cas particulier où $\overrightarrow{\mathrm{A}}^{\prime}=\overrightarrow{\mathrm{A}}$, la relation (12) devient : 


$$
\frac{1}{\mathrm{~V}} \int_{\Omega} \vec{\nabla} \mathrm{p} \cdot \mathbf{k} \cdot \vec{\nabla} \mathrm{p} \mathrm{dv}=\overrightarrow{\mathrm{A}} \cdot \mathbf{K}_{\Omega} \cdot \overrightarrow{\mathrm{A}}
$$

Comme $\mathbf{k}$ est positif, le premier et donc le second membre de (13) sont toujours positifs, ce qui montre que $\mathbf{K}_{\Omega}$ est positif. Si un vecteur $\overrightarrow{\mathrm{A}}$ annule le second membre, le $\vec{\nabla} \mathrm{p}$ correspondant doit être nul au premier membre (car $\mathbf{k}$ est défini), et donc sa moyenne $\overrightarrow{\mathrm{G}}$ est également nulle. On déduit de alors (9) que $\overrightarrow{\mathrm{A}}$ est nulle, ce qui montre que le tenseur $\mathbf{K}_{\Omega}$ est défini.

En reportant (9) dans (11) on trouve :

$$
\overrightarrow{\mathrm{Q}}=-\mathbf{K}_{\Omega} \cdot \overrightarrow{\mathrm{G}}
$$

Le tenseur $\mathbf{K}_{\Omega}$ reliant le flux moyen au gradient moyen de pression dans le domaine $\Omega$ (sous conditions de pression linéaire au contour) sera appelé le tenseur de conductivité hydraulique moyenne de ce domaine. Nous venons de montrer qu'il est symétrique et défini-positif. Par ailleurs, en reportant (9) dans (13) on trouve :

$$
\frac{1}{\mathrm{~V}} \int_{\Omega} \vec{\nabla} \mathrm{p} \cdot \mathbf{k} \cdot \vec{\nabla} \mathrm{p} \mathrm{dv}=\overrightarrow{\mathrm{G}} \cdot \mathbf{K}_{\Omega} \cdot \overrightarrow{\mathrm{G}}
$$

Au premier membre de (15) nous trouvons l'expression de la dissipation donnée par Indelman et Dagan [11]. Cette relation montre que la dissipation macroscopique calculée par $\mathbf{K}_{\Omega}$ est égale à la dissipation moyenne réelle dans le milieu (sous conditions de pression linéaire au contour).

Supposons maintenant que $\Omega$ soit un sous-domaine d'un milieu hétérogène infini et qu'il soit caractérisé par une longueur caractéristique D (par exemple le diamètre moyen). Supposons que pour le milieu considéré, $\mathbf{K}_{\Omega}$ tende vers une limite $\mathbf{K}$ quand $\mathbf{D}$ tend vers l'infini. Si la limite $\mathbf{K}$ existe, elle est symétrique et positive. Dans ce cas, le comportement à grande échelle du milieu considéré peut être assimilé à un milieu poreux de perméabilité $\mathbf{K}$.

\section{Application aux milieux fracturés}

Dans le cas d'un milieu fracturé bi-dimensionnel, la formule (6) s'écrit sous la forme discrétisée :

$$
\overrightarrow{\mathrm{Q}}=\frac{1}{\mathrm{~V}} \sum_{\kappa} \mathrm{q}^{(\mathrm{\kappa})} \overrightarrow{\mathrm{x}}^{(\kappa)}
$$

où $\overrightarrow{\mathrm{x}}^{(\kappa)}$ est le vecteur position d'un point d'intersection des fractures avec le contour de $\mathrm{du}$ domaine et $\mathrm{q}^{(\mathrm{\kappa})}$, le flux sortant de la fracture en ce point. En calculant ce vecteur pour deux directions différentes de $\overrightarrow{\mathrm{A}}$, on construit $\mathbf{K}_{\Omega}$. Cette méthode apporte une grande simplification par rapport à celles utilisées dans [1] et [3] qui passent par la construction d'un diagramme de perméabilité directionnelle. Pour illustrer ces résultats, nous avons considéré un carré $\mathrm{ABCD}$ dans un milieu fracturé (figure 1a). Le diagramme $1 / \sqrt{\mathrm{K}_{\mathrm{g}}}$ calculé pour ce domaine par la méthode [1], c'est-à-dire par des rotations successives du carré par rapport au réseau de fractures (qui couvre un domaine plus grand que $A B C D$ ) 
est donné dans la figure $1 \mathrm{~b}$. Nous avons calculé un autre diagramme en faisant les mêmes rotations mais en calculant chaque fois le flux $\vec{Q}$ par la formule (16) et en la projetant sur la direction du gradient pour avoir la perméabilité directionnelle. On voit que les points obtenus de cette manière s'alignent presque sur une ellipse. La grande dispersion des points du premier diagramme est due au fait que le flux calculé par la méthode [1] ne représente pas correctement le flux moyen dans le domaine. Nous avons calculé également les $\mathbf{K}_{\boldsymbol{\Omega}}$ du carré $\mathrm{ABCD}$ et du domaine polygonal EFGHIJKLMNL par notre méthode. Pour comparer les résultats, nous avons représenté graphiquement ces deux tenseurs par les ellipses correspondantes. Ces ellipses et le deuxième diagramme se superposent presque, ce qui indique qu'à l'échelle d'hétérogénéité considérée (l'échelle des fractures), les domaines étudiés sont de taille suffisamment grande par rapport à la taille du Volume Elémentaire Représentatif, et que le $\mathbf{K}_{\Omega}$ ainsi obtenu est très proche de la perméabilité effective du milieu.

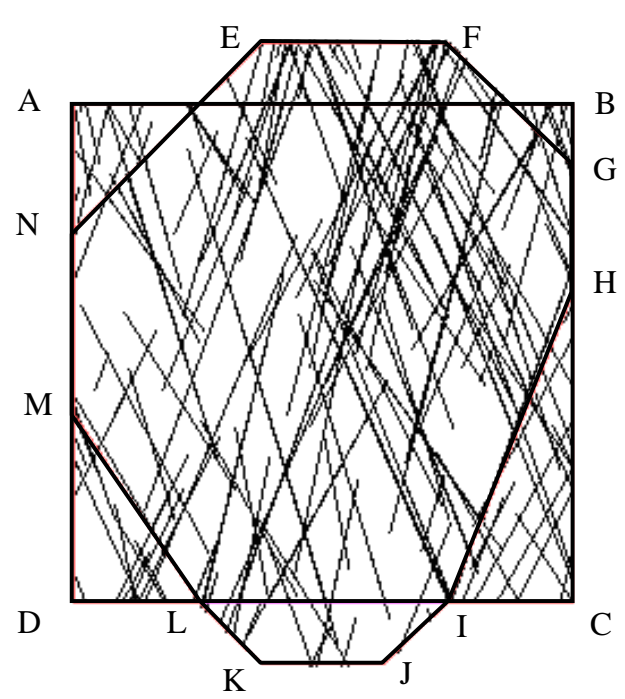

(a)

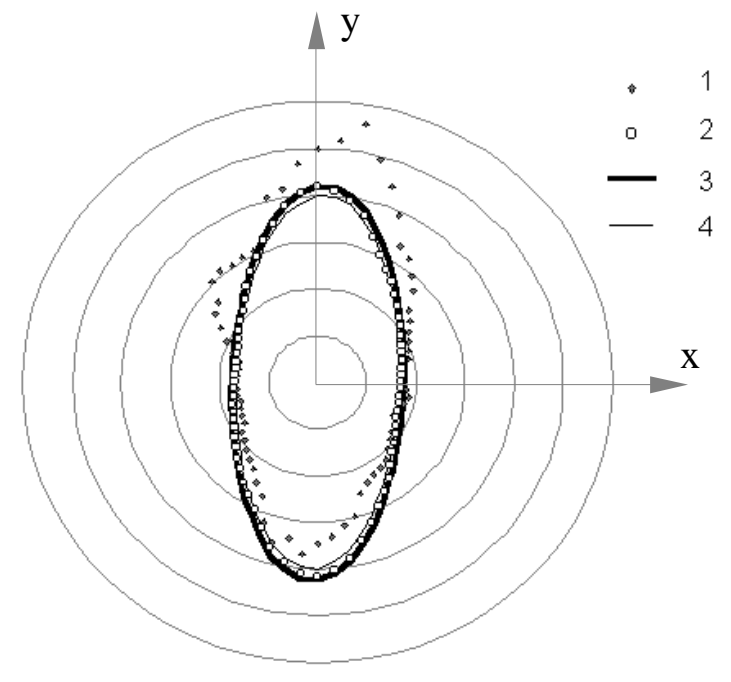

(b)

Figure 1 - a : Domaines étudiés, b : Diagramme obtenu par la méthode [1] en partant du carré $\mathrm{ABCD}$ (1), diagramme obtenu par la même méthode mais en utilisant l'expression (16) pour le flux moyen (2), ellipses représentant les tenseurs $\mathbf{K}_{\boldsymbol{\Omega}}$ du domaine ABCD (3) et EFGHIJKLMN (4).

Figure 1 - a : Studied domains, b : Diagram obtained by the method [1] beginning on the square $\mathrm{ABCD}$ (1), diagram obtained by the same method but using the expression (16) for the mean flux (2), ellipses representing $\mathbf{K}_{\boldsymbol{\Omega}}$ tensors for the domains $\mathrm{ABCD}$ (3) and EFGHIJKLMN (4). 


\section{Conclusions}

La perméabilité équivalente d'un domaine hétérogène et de forme quelconque peut être étudiée par une méthode non locale en appliquant sur la frontière de ce domaine des conditions de pression linéaire au contour définies par la relation (7). Le gradient moyen de pression dans le domaine sera alors exactement égal à $\vec{A}$. Le flux moyen peut être plus facilement calculé à partir des valeurs de flux sur la frontière par la formule (6). La relation entre ce flux moyen et $\overrightarrow{\mathrm{A}}$ est linéaire et définit un tenseur de conductivité hydraulique moyenne pour le domaine. Ce tenseur peut être identifié en calculant le flux moyen pour deux ou trois (suivant la dimension du problème) directions différentes de $\overrightarrow{\mathrm{A}}$. Nous avons montré que ce tenseur est symétrique et défini positif si en tout point du domaine, le tenseur local de perméabilité est symétrique et défini positif.

\section{Références}

[1] Long J.C.S., Remer J.S., Wilson C.R., Witherspoon P.A, "Porous media equivalents for networks of discontinuous fractures", Water Resources Research, vol. 18, NO. 3, (1982), pp. 645-658.

[2] Schwartz F.W., Smith L., "A continuum approach for modelling mass transport in fractured media", Water Resources Research, vol. 24, NO. 8, (1988), pp. 1360-1372.

[3] Cacas M.C., Ledoux E., de Marsily G., Tillie B., «Modeling fracture flow with a stochastic discrete fracture network : calibration and validation, 1. The flow model", Water Resources Research, vol. 26, NO. 3, (1990), pp. 479-489.

[4] Fidelibus C., Barla G., Cravero M., "Alternative schemes for the assessment of the equivalent continuum hydraulic properties of rock masses", Eurock '96, Barla (ed.), Balkema, Rotterdam, (1996), pp. 1243-1252.

[5] Renard Ph. and de Marsily G., "Calculating equivalent permeability : a review", Advances in Water Resources, vol. 20, NOs. 5-6, (1997), pp. 253-278.

[6] Sánchez-Vila X., Girardi G.P., Carrera J., "A synthesis of approaches to upscaling of hydraulic conductivities", Water Resources Research, vol. 31, NO. 4, (1995), pp. 867882.

[7] Goméz-Hernández, J.J. and Wen X.H., "Upscaling hydraulic conductivities in heterogeneous media : an overview", Journal of Hydrology, 183 (1996), pp. ix-xxxii.

[8] Nœtinger B., "Computing the effective permeability of log-normal permeability fields using renormalization methods", C.R. Acad. Sci. Paris, Sciences de la Terre et des Planètes/Earth and Planetary Sciences 331 (2000) 353-357.

[9] Rubin, Y. and Goméz-Hernández, J.J.,"A stochastic approach to the problem of upscaling of conductivity in disordered media : theory and unconditional numerical simulations", Water Resources Research, vol. 26, NO. 4, (1990), pp. 691-701.

[10] Zaoui A., "Structural morphology and constitutive behaviour of microheterogeneous materials", Continuum micromechanics, CISM courses and lectures $n^{\circ} 377$, P. Suquet, ed., Springer, New York.

[11] Indelman P. and Dagan G., "Upscaling of permeability of anisotropic heterogeneous formations, 1.The general framework", Water Resources Research, vol. 29, NO. 4, (1993), pp. 917-923. 